\section{Duração do aleitamento materno em menores de dois anos de idade em Itupeva, São Paulo, Brasil: há diferenças entre os grupos sociais?}

\section{Breast feeding duration in children under two years of age in Itupeva, São Paulo, Brazil: are there differences among social groups?}

Elizabeth Fujimori 1

Áurea Tamami Minagawa 2

Daniela Laurenti 3

Rosali Maria Juliano Marcondes Montero 4

Ana Luiza Vilela Borges 5

Ida Maria Vianna de Oliveira 6

1,3-6 Departamento de Enfermagem em Saúde Coletiva. Escola de Enfermagem. Universidade de São Paulo. Av. Dr. Enéas de Carvalho Aguiar, 419. São Paulo, SP, Brasil. CEP 05403-000. E-mail: efujimor@usp.br

2 Curso de Graduação em Enfermagem. Departamento de Saúde da Criança, Adolescente e Mulher. Faculdade de Ciências Médicas da Santa Casa de São Paulo. São Paulo, SP, Brasil.

\section{Resumo}

Objetivos: conhecer a duração do aleitamento

Objective: based on the theory of social determination of the health-disease process, the objective of this study was to know the duration of overall breast feeding and the factors associated with its interruption, according to the social reproduction profiles of the families (ways of living and working).

Methods: this is a cross-sectional study carried out with a representative sample of 261 children under two years of age, living in Itupeva city, Sao Paulo, Brazil. From a theoretical-methodologicaloperational basis, three social homogeneous groups (GSH) were established, according to similar conditions of working and living. Overall breast feeding duration was calculated from survival tables. Variables with $p<0.20$ in bivariate Wilcoxon test were then introduced in multiple Cox Regression model in order to find associated aspects to the breast feeding duration.

Results: the breast feeding duration medians of the 3 GSH were 6.7 months, 7.1 months and 9.9 months, with no statistically significant difference $(p=0.31)$. The multivariate analysis showed that the sequence of birth $(p=0.018)$, pacifier use $(p<0.001)$ and bottle-feeding $(p<0.001)$ were associated to the interruption of breast feeding.

Conclusions: there was no association between breast feeding duration and the social groups studied. However, the social determinants on breast feeding duration should be considered in actions that promote breast feeding. These actions also need to include information about harmful effects to the duration of breastfeeding when offering pacifiers and bottle-feedings.

Key words Breast feeding, Social group, Social conditions materno (AM) total e os determinantes associados à sua interrupção segundo perfis de reprodução social das familias (formas de trabalhar e viver), fundamentados na teoria da determinação social do processo saúde-doença.

Métodos: estudo transversal conduzido em uma amostra de 261 crianças menores de dois anos, residentes em Itupeva, São Paulo, Brasil. A partir de uma base teórico-metodológica-operacional compuseramse três grupos sociais homogêneos (GSH) segundo semelhantes formas de trabalhar $e$ de viver. A duração do AM total foi estimada com a técnica de tábuas de vida. O teste de Wilcoxon foi empregado para identificar associação entre as variáveis categóricas na análise bivariada. Para análise múltipla, as variáveis associadas com a duração mediana do $A M(p<0,20)$ foram inseridas num modelo de Regressão de Cox.

Resultados: as medianas de aleitamento materno para os $3 \mathrm{GSH}$ foram 6,7 meses, 7,1 meses e 9,9 meses, sem diferença estatística significante $(p=0,31)$. O modelo de análise múltipla selecionou como significante as variáveis: ordem de nascimento $(p=0,018)$, uso de chupeta $(p<0,001)$ e uso de mamadeira $(p<0,001)$.

Conclusões: não foi constatada associação entre duração do AM e os grupos sociais estudados. Entretanto, não há como se desconsiderar a relevância dos determinantes sociais nas ações de promoção do AM, que necessariamente devem incluir esclarecimentos sobre os efeitos prejudiciais do hábito de oferecer chupetas e mamadeiras.

Palavras-chave Aleitamento materno, Grupo social, Condições sociais 


\section{Introdução}

As vantagens da amamentação para a saúde da criança têm sido reiteradas em diferentes realidades sócio-culturais, pois sua prática contribui para redução da incidência e gravidade de uma série de morbidades, tais como diarréia, infecções respiratórias, infecção urinária, enterocolite necrotizante entre outras. 1 Também há evidências de que o leite materno pode proteger a criança contra a síndrome da morte súbita, diabetes mellitus insulino-dependente e não insulino-dependente, sobrepeso e obesidade, hipercolesterolemia e asma. ${ }^{1}$

Apesar desses resultados e da unanimidade dos estudos em atestar que o leite materno é o melhor alimento para o lactente, a prevalência e duração do aleitamento materno variam de acordo com a época e os diferentes contextos sócio-culturais. ${ }^{2}$ Em países desenvolvidos há estudos que continuam a evidenciar que mulheres pobres amamentam menos e por períodos mais curtos. ${ }^{3}$ No Brasil, os dados disponíveis são bastante heterogêneos e sua comparabilidade é limitada, pois além dos estudos utilizarem metodologias diferentes, são conduzidos em contextos sócio-culturais e históricos bastante diversos. Há evidências de que o baixo nível socioeconômico não se associa com a interrupção precoce do aleitamento materno (AM). ${ }^{4}$ No entanto, outros estudos indicam a existência de associação entre baixas condições de vida e frequência e duração do AM.5,6 Assim, registra-se conflito entre esses eventos.

Estudo da década de $1980^{7}$ destacava como fatores mais frequentemente relacionados à prática do aleitamento materno: níveis socioeconômico, educacional, idade da mãe, distribuição urbanorural, paridade, trabalho da mulher, início da primeira mamada, introdução de alimentação complementar, ocupação do pai e condições de saúde da mãe. Para Buralli, ${ }^{7}$ havia predominância do modelo biológico e individual nas explicações sobre a prática do aleitamento materno, apesar da aparente relevância dada ao social.

Recente revisão ${ }^{8}$ de estudos publicados entre 1990 e 2004, analisando os aspectos que mais frequentemente influenciam a decisão de amamentar, a duração da amamentação e as razões do desmame, indica a persistência dos mesmos fatores, de forma que baixo nível socioeconômico e educacional maternos, maternidade precoce $\mathrm{e}$ trabalho fora do lar ainda atuam como fatores de risco para a menor duração do aleitamento materno.

O pressuposto de que a opção materna de amamentar envolve a interação de aspectos sociais, econômicos, políticos, culturais e psicológicos, que transformam um ato aceito como natural e fisiológico em um ato histórico, regulável pela sociedade, tem sido utilizado na tentativa de justificar diferentes práticas da amamentação. ${ }^{9} \mathrm{O}$ campo da saúde coletiva considera o processo saúde-doença como socialmente determinado, de forma que "a articulação entre o social e o biológico se dá pela forma como cada grupo social se insere no mundo do trabalho e no mundo da vida". 10 Segundo essa teoria, as condições de trabalho e vida imprimem desgaste ou fortalecimento dos indivíduos, expondo-os a diferentes riscos de adoecer e morrer. Assim, a categoria proposta para estudar e explicar como o social interfere no processo saúde-doença se relaciona à reprodução social, a qual diz respeito tanto às condições de trabalho quanto às condições de vida.

O presente trabalho, estruturado sob o marco teórico da determinação social do processo saúdedoença, teve como objetivo conhecer a duração total do aleitamento materno e os determinantes associados à sua interrupção, na perspectiva diferenciada dos perfis de reprodução social.

\section{Métodos}

Esse é um estudo transversal que integrou um projeto mais amplo desenvolvido no município de Itupeva, SP, cujos resultados sobre a prevalência das diferentes categorias do aleitamento materno (aleitamento materno exclusivo, predominante, complementado em tempo oportuno contínuo 1 e 2 ) foram publicados em outro artigo. 11

A amostra do projeto maior foi delineada para ser representativa e proporcional ao número de crianças menores de dois anos de idade, residentes na zona urbana do município. Para garantir a representatividade da amostra para os diversos subprojetos, além da estimativa de 976 crianças menores de dois anos 12 e do número de domicílios na área urbana do município, 13 considerou-se a prevalência estimada de $45 \%$ de anemia, valor próximo ao obtido na cidade de São Paulo para crianças de zero a 59 meses de idade. $14 \mathrm{O}$ tamanho da amostra foi calculado utilizando-se um nível de significância de 5\%, com poder de $80 \%$ e margem de erro de $5 \%$. Também foi considerada uma previsão de $10 \%$ de perdas e recusas, o que resultou em um tamanho amostral planejado de 274 crianças menores de dois anos de idade. Mesmo que a amostra não tenha sido calculada especificamente para avaliar a relação estudada, é importante salientar que a prevalência de AM no momento da entrevista $(41 \%)^{11}$ foi próximo à prevalência de $45 \%$ de anemia tomada como base 
para o cálculo. Utilizou-se o procedimento de amostragem por conglomerados com sorteio em três etapas: setores censitários urbanos, seguidos de quadras e domicílios. A amostra final foi constituída por 261 crianças.

A pesquisa foi aprovada pelo Comitê de Ética em Pesquisa da Escola de Enfermagem da Universidade de São Paulo (CEP EEUSP), sob o Processo $n^{\circ}$ $156 / 2001$ e os dados foram obtidos por inquérito domiciliar, realizado em julho e agosto de 2001, por equipe treinada. As entrevistas foram realizadas somente com a mãe da criança, utilizando-se um formulário pré-testado com questões objetivas e respostas fechadas, relacionadas às características familiares (renda familiar per capita e escolaridade do pai); características maternas (idade, escolaridade, inserção no trabalho, situação conjugal, número de filhos, orientações sobre AM no pré-natal e no parto); características infantis (idade, sexo, peso ao nascer, tempo e ordem de nascimento, uso de mamadeira e de chupeta), além da prática alimentar, aleitamento materno e época de introdução da alimentação complementar no esquema alimentar da criança.

Para atender ao objetivo desse estudo, optou-se por identificar os processos determinantes da duração do AM total por meio de indicadores que, explicitassem os perfis de reprodução social das famílias a que pertenciam as crianças estudadas. Para caracterizar as formas de reprodução social neste trabalho, utilizou-se, com modificações, uma base teórico-metodológica-operacional15 que, a partir de indicadores compostos, permitiu construir três diferentes agrupamentos sociais com condições homogêneas de trabalhar e viver. As formas de trabalhar (FT) foram dimensionadas por três variáveis: renda familiar per capita (RFPC), registro formal no trabalho e usufruto de benefícios decorrentes da inserção no trabalho, e as formas de viver (FV) por outras três variáveis: propriedade da habitação, atividades de lazer e grau de agregação social - participação em grupos/associações da comunidade ou sindicatos.

Consideraram-se formas de trabalhar estáveis, a presença, na família, de pelo menos duas das seguintes situações: RFPC $\geq 1,8$ salários mínimos, um membro com registro em carteira profissional e/ou com usufruto (possuir e desfrutar) de dois ou mais benefícios, sendo um dos benefícios relacionados à saúde. Com as formas de viver estáveis, consideraram-se famílias com pelo menos duas das seguintes situações: proprietária da habitação, um membro da família com atividade de lazer não relacionada à TV/vídeo e/ou participação em associ- ações. Famílias que não atingiram esse limite foram classificadas com FT instáveis e/ou FV instáveis. Com a aplicação desse processo, as famílias foram agrupadas em três diferentes grupos sociais homogêneos (GSH): GSH1, que incluiu famílias com FT e FV estáveis (melhor inserção social); GSH2, que congregou famílias com FT estáveis e FV instáveis ou vice-versa (inserção social intermediária); e GSH3, formado por famílias com FT e FV instáveis (pior inserção social). ${ }^{16}$

Foram consideradas em AM todas as crianças alimentadas com leite materno, sendo a duração do AM total definida como o tempo em que a criança recebeu leite materno, independente do consumo de qualquer complemento, lácteo ou não. ${ }^{17}$

Para as análises estatísticas, utilizaram-se os pacotes estatísticos EpiInfo 6 e SPSS 8.0. A mediana do AM foi obtida com a técnica da análise de sobrevida, considerando-se como evento final a interrupção definitiva da amamentação, isto é, o tempo do AM foi censurado a partir dos 24 meses, inclusive. O teste do qui-quadrado ou exato de Fisher, foram usados para identificar associação entre as variáveis infantis, maternas, sócio-demográficas e os diferentes GSH. Análise bivariada avaliou, inicialmente, associação entre a duração do AM (variável dependente) e o GSH e outras variáveis independentes, por meio do teste de Wilcoxon. Adotou-se um nível de significância de 5\%. Para evitar a exclusão de variáveis potencialmente importantes para a associação investigada, as variáveis que apresentaram associação, na análise bivariada, com o tempo de AM no nível de significância de $20 \%$ $(p<0,20)$ foram incluídas no modelo de Regressão de Cox, com processo de seleção de variáveis backward. As variáveis com mais de duas categorias foram transformadas em dummies. Os resultados foram expressos em razão de densidade de incidências (hazard ratio - HR), com intervalo de confiança (IC) de $95 \%$ e p-valor com nível de significância de $5 \%(p<0,05)$.

\section{Resultados}

A caracterização dos perfis de reprodução social indicou que $19,2 \%(n=50)$ das crianças pertenciam a famílias do GSH1; 36,4\% (n=95) a famílias do GSH2 e 44,4\% (n=116) a famílias do GSH3.

$\mathrm{Na}$ Tabela 1, apresentam-se algumas características infantis, maternas (pré-natal e parto) e sóciodemográficas segundo os GSH, resultantes das análises bivariadas. Constatou-se que, no geral, predominaram crianças com menos de um ano, do sexo masculino; porém, a distribuição quanto ao 
Distribuição de algumas características infantis, maternas (pré-natal e parto) e sócio-demográficas por Grupos Sociais Homogêneos. Itupeva, SP, 2001.

Variáveis

Grupos Sociais Homogêneos*

Total

\begin{tabular}{lll}
\hline GSH1 & GSH2 & GSH3 \\
\hline
\end{tabular}

\begin{tabular}{|c|c|c|c|c|c|c|c|c|}
\hline & & & & & & & & \\
\hline & $\mathrm{n}$ & $\%$ & $\mathrm{n}$ & $\%$ & $\mathrm{n}$ & $\%$ & $\mathrm{n}$ & $\%$ \\
\hline Total de crianças & 50 & 100,0 & 95 & 100,0 & 116 & 100,0 & 261 & 100,0 \\
\hline \multicolumn{9}{|l|}{ Infantis } \\
\hline \multicolumn{9}{|l|}{ Faixa etária (meses) } \\
\hline $0 \mid-6$ & 12 & $24,0 \mathrm{a}$ & 23 & $24,2 \mathrm{a}$ & 38 & $32,8 \mathrm{a}$ & 73 & 27,9 \\
\hline $6 \mid-12$ & 11 & $22,0 a$ & 22 & $23,2 \mathrm{a}$ & 35 & $30,1 \mathrm{a}$ & 35 & 13,4 \\
\hline $12 \mid-18$ & 15 & $30,0 a$ & 29 & $30,5^{a}$ & 22 & $19,0 \mathrm{a}$ & 36 & 13,8 \\
\hline $18 \mid-24$ & 12 & $24,0 a$ & 21 & $22,1 \mathrm{a}$ & 21 & $18,1 \mathrm{a}$ & 32 & 12,3 \\
\hline Sexo masculino & 31 & $62,0 a$ & 54 & $56,8^{a}$ & 56 & $48,3 a$ & 141 & 54,0 \\
\hline Peso ao nascer $\leq 2500 \mathrm{~g} * *$ & 5 & $10,2^{a}$ & 7 & $7,5^{a}$ & 6 & $5,2^{a}$ & 18 & 6,9 \\
\hline Nascimento pré-termo & 3 & $6,0^{a}$ & 5 & $5,3 a$ & 14 & $12,1^{a}$ & 22 & 8,4 \\
\hline \multicolumn{9}{|l|}{ Ordem de nascimento } \\
\hline $1^{\circ}$ filho & 30 & $60,0^{a}$ & 29 & $30,5^{b}$ & 40 & $34,5^{b}$ & 99 & 37,9 \\
\hline $2^{\circ}$ filho & 18 & $36,0^{a}$ & 39 & $41,1^{a}$ & 34 & $29,3^{a}$ & 91 & 34,9 \\
\hline $3^{\circ}$ filho em diante & 2 & $4,0 a$ & 27 & $28,4 b$ & 42 & $36,2 c$ & 71 & 27,2 \\
\hline Uso de mamadeira & 39 & $78,0^{a}$ & 81 & $85,3 a$ & 86 & $74,1 \mathrm{a}$ & 206 & 78,9 \\
\hline Uso de chupeta & 29 & $58,0 a$ & 54 & $56,8^{a}$ & 56 & $48,3 a$ & 139 & 53,3 \\
\hline \multicolumn{9}{|l|}{ Pré-natal } \\
\hline Atendimento pré-natal & 50 & $100,0 \mathrm{a}$ & 95 & $100,0 a$ & 115 & $99,1 \mathrm{a}$ & 260 & 99,6 \\
\hline $\begin{array}{l}\text { Atendimento em } \\
\text { serviço público }\end{array}$ & 22 & $44,0 a$ & 64 & $67,4 b$ & 99 & $86,1 c$ & 185 & 71,2 \\
\hline Número de filhos ( $\geq 3$ filhos) & 2 & $4,0 \mathrm{a}$ & 27 & $28,4 b$ & 44 & $37,9 c$ & 73 & 28,0 \\
\hline Orientação sobre AM & 28 & $56,0 a$ & 51 & $53,7 a$ & 52 & $44,8 \mathrm{a}$ & 131 & 50,2 \\
\hline \multicolumn{9}{|l|}{ Parto } \\
\hline Cesárea & 24 & $48,0^{a}$ & 33 & $34,7^{a}$ & 30 & $25,9 \mathrm{~b}$ & 87 & 33,3 \\
\hline Em serviço público & 26 & $52,0^{a}$ & 65 & $68,4 b$ & 98 & $84,5 c$ & 189 & 72,4 \\
\hline Orientação sobre AM & 45 & $90,0^{a}$ & 83 & $87,4^{a}$ & 98 & $84,5^{a}$ & 226 & 86,6 \\
\hline \multicolumn{9}{|l|}{ Características sociodemográficas } \\
\hline $\mathrm{RFPC} \geq 1,8 \mathrm{SM}$ & 22 & $44,0^{a}$ & 19 & $20,0^{b}$ & 5 & $4,3 c$ & 46 & 17,6 \\
\hline Idade materna (<30 anos) & 42 & $84,0 a$ & 60 & $63,2 b$ & 83 & $71,6 \mathrm{~b}$ & 185 & 70,9 \\
\hline \multicolumn{9}{|l|}{ Escolaridade materna } \\
\hline \multicolumn{9}{|l|}{ (anos de estudo) } \\
\hline Até 3 & 2 & $4,0 a$ & 7 & $7,4 a$ & 19 & $16,4 b$ & 28 & 10,7 \\
\hline 4 a 11 & 45 & $90,0 a$ & 83 & $87,4 a$ & 93 & $80,2 a$ & 221 & 84,7 \\
\hline 12 e mais & 3 & $6,0 a$ & 5 & $5,2 a$ & 4 & $3,4 a$ & 12 & 4,6 \\
\hline Mãe sem companheiro & 14 & $28,0 a$ & 13 & $13,7 b$ & 10 & $9,6 c$ & 37 & 14,2 \\
\hline Trabalho regular materno & 21 & $42,0 a$ & 29 & $30,5 a$ & 24 & $20,7 \mathrm{~b}$ & 131 & 50,2 \\
\hline \multirow{2}{*}{\multicolumn{9}{|c|}{$\begin{array}{l}\text { Escolaridade do pai } \\
\text { (anos de estudo)** }\end{array}$}} \\
\hline & & & & & & & & \\
\hline Até 3 & 0 & $0,0 \mathrm{a}$ & 3 & $3,8^{a}$ & 11 & $10,9 b$ & 14 & 6,5 \\
\hline 4 a 11 & 29 & $82,9 a$ & 73 & $93,6^{a}$ & 87 & $86,1^{a}$ & 189 & 88,3 \\
\hline 12 e mais & 6 & $17,1 \mathrm{a}$ & 2 & $2,6 b$ & 3 & $3,0 \mathrm{~b}$ & 11 & 5,2 \\
\hline
\end{tabular}

*Para cada variável, as distribuições percentuais nas colunas seguidas de letras iguais não diferiram significantemente $(p>0,05)$ e letras diferentes indicam que houve diferença entre os GSHs $\left(p<0,05\right.$; teste do $\chi^{2}$ ou teste exato de Fisher); **Não se obteve dados para todos; GSH=grupos sociais homogêneos; $A M=$ aleitamento materno; RFPC= renda familiar per capita; $\mathrm{SM}=$ salário mínimo. 
sexo e idade foi homogênea entre os três grupos, da mesma forma que o percentual de baixo peso e nascimentos pré-termo. O GSH1 apresentou concentração significantemente maior de $1^{\circ}$ filho e mães com menor número de filhos, enquanto os grupos mais precariamente incluídos apresentaram maior proporção de $3^{\circ}$ filho em diante e mães com maior número de filhos $(p<0,05)$. Uso de chupeta $(53,3 \%)$ e mamadeira $(78,9 \%)$ foram elevados nos três GSH, não se constatando diferença estatisticamente significante entre os mesmos. O pré-natal foi realizado praticamente pela totalidade das mães, com diferença estatisticamente significante $(p<0,05)$ apenas em relação ao local da atenção, sendo os serviços públicos mais utilizados pelos grupos sociais mais excluídos. O mesmo se verificou em relação ao local de realização do parto. Todas as variáveis sóciodemográficas analisadas apresentaram diferença estatisticamente significante entre os GSH $(p<0,05)$.

No momento da entrevista, $32,0 \%$ das crianças do GSH1 ainda eram amamentadas, proporção que no GSH2 era de $36,8 \%$ e no GSH3 de $48,3 \%$, percentual mais elevado em relação aos demais grupos, porém sem diferença estatística significante $(p=0,09)$.

Os resultados apresentados na Figura 1 mostraram que a duração do AM em Itupeva se relacionava com os perfis de reprodução social expressos nos GSH. Constatou-se que as curvas da duração do aleitamento materno não diferiram de forma estatisticamente significante $(p=0,31)$; no entanto, observou-se discreta vantagem para as crianças do GSH3: a mediana de duração do AM aumentou do GSH1 para GSH2 e deste para o GSH3, passando de 6,7 meses para 7,1 e 9,9 meses, respectivamente. A tendência de manutenção mais prolongada do AM no GSH3, em relação aos demais grupos, pôde ser justificada e melhor entendida quando se analisou o comportamento das diferentes variáveis que caracterizavam as mães e as crianças nos GSH, concomitante à análise do conjunto de variáveis que explicitam o padrão de reprodução social.

\section{Figura 1}

Curvas de sobrevivência do aleitamento materno segundo Grupos Sociais Homogêneos. Itupeva, SP, 2001.

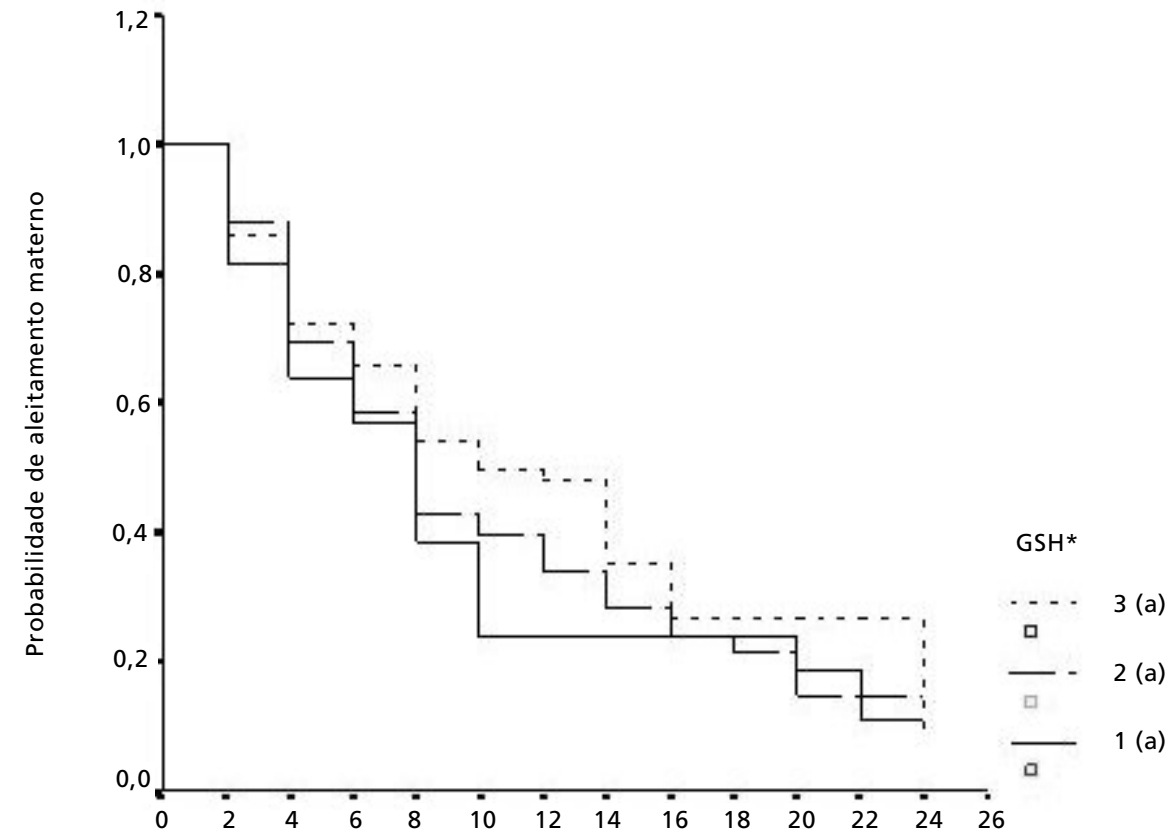

Tempo (meses) de aleitamento materno

GSH=grupos sociais homogêneos; *Para cada variável, as faixas seguidas de letras iguais não diferiram significativamente ( $p=0,31$; teste de Wilcoxon). 
Assim, para avaliar como a duração do AM em Itupeva se relacionava com os perfis de reprodução social - expressos nos GSH - as características estudadas (exceto idade) foram submetidas à análise bivariada utilizando-se o teste de Wilcoxon, considerando-se como variável dependente o tempo de AM, obtido por meio da técnica da análise de sobrevida, nos diferentes GSH (Tabela 2). Observouse que duas variáveis mostraram associação com o tempo de AM nos três GSH: uso de chupeta e de mamadeira. As outras variáveis que também afetaram significantemente a duração do AM nos diferentes GSH foram: RFPC no GSH2 e ordem de nascimento, número de filhos e escolaridade do pai no GSH3. A RFPC $\geq 1,8$ salário mínimo associou-se com maior tempo de AM no GSH2, ou seja, maior renda comportou-se como protetor do AM nesse grupo $(p=0,04)$, de forma que nas famílias com RFPC $\geq 1,8$ salário mínimo, a mediana de sobrevida do AM foi de 7,2 meses, enquanto para aquelas com RFPC $<1,8$ salário mínimo, a mediana foi de 4,4 meses. No GSH3, a ordem de nascimento interferiu com significância $(p<0,02)$ na curva de sobrevida do AM, com mediana de duração do AM de 4,9 meses para as primogênitas e 12,0 meses para as crianças de segunda ordem em diante. O maior número de filhos também representou mais tempo de AM para as mulheres do GSH3 $(p=0,02)$. Ainda no GSH3, a escolaridade do pai interferiu na duração do AM, mas não de forma linear. Crianças cujos pais tinham menos de três anos de estudo apresentaram mediana de duração do AM de 24 meses; a mediana para aquelas cujos pais estudaram de 4 a 11 anos foi de 7,6 meses; enquanto as crianças de pais com 12 anos ou mais de estudo apresentaram mediana de 19 meses $(p<0,01)$.

As variáveis que apresentaram nível de significância de $20 \%(p<0,20)$ em qualquer grupo e/ou no total da amostra foram inseridas no modelo de Regressão de Cox para a análise múltipla. A variável GSH também foi inserida no modelo, apesar de não apresentar associação com o tempo de AM na análise bivariada $(p>0,20)$. Permaneceram significantemente associadas à duração do aleitamento materno, apenas as variáveis: ordem de nascimento da segunda posição em diante, que se mostrou protetora $(p=0,018)$, uso de mamadeira $(p<0,001)$ e uso de chupeta $(p<0,001)$, conforme mostram os resultados apresentados na Tabela 3 .

Tabela 2

Mediana de tempo de aleitamento materno (meses) nos diferentes Grupos Sociais Homogêneos, segundo características infantis, maternas (pré-natal e parto) e sócio-demográficas familiares. Itupeva, SP, 2001.

\begin{tabular}{|c|c|c|c|c|}
\hline \multirow[t]{2}{*}{ Variáveis } & \multicolumn{3}{|c|}{ Grupos Sociais Homogêneos } & \multirow[t]{2}{*}{ Total } \\
\hline & GSH1 & GSH2 & GSH3 & \\
\hline Aleitamento materno (Mediana, meses) & 6,7 & 7,1 & 9,9 & 7,2 \\
\hline \multicolumn{5}{|l|}{ Características infantis } \\
\hline \multicolumn{5}{|l|}{ Sexo } \\
\hline Masculino & 6,11 & 6,87 & 9,83 & 7,28 \\
\hline Feminino & 7,54 & 6,56 & 11,19 & 7,89 \\
\hline$p^{*}$ & 0,779 & 0,437 & 0,940 & 0,485 \\
\hline \multicolumn{5}{|l|}{ Peso ao nascer } \\
\hline Baixo peso & 6,75 & 16,15 & 22,00 & 7,28 \\
\hline Peso normal & 6,62 & 6,77 & 11,24 & 7,63 \\
\hline$p$ & 0,655 & 0,694 & 0,221 & 0,723 \\
\hline \multicolumn{5}{|l|}{ Tempo de nascimento } \\
\hline Termo & 6,37 & 6,84 & 11,33 & 7,62 \\
\hline Pré-termo & 18,25 & 4,42 & 6,78 & 7,11 \\
\hline$p$ & 0,162 & 0,319 & 0,338 & 0,609 \\
\hline \multicolumn{5}{|l|}{ Ordem de nascimento } \\
\hline $1^{\circ}$ & 6,51 & 5,40 & 4,87 & 6,12 \\
\hline $2^{\circ}$ em diante & 6,82 & 7,53 & 12,04 & 8,68 \\
\hline$p$ & 0,906 & 0,138 & 0,021 & 0,006 \\
\hline
\end{tabular}


continuação

Tabela 2

Mediana de tempo de aleitamento materno (meses) nos diferentes Grupos Sociais Homogêneos, segundo características infantis, maternas (pré-natal e parto) e sócio-demográficas familiares. Itupeva, SP, 2001.

\begin{tabular}{|c|c|c|c|c|}
\hline \multirow[t]{2}{*}{ Variáveis } & \multicolumn{3}{|c|}{ Grupos Sociais Homogêneos } & \multirow[t]{2}{*}{ Total } \\
\hline & GSH1 & GSH2 & GSH3 & \\
\hline \multicolumn{5}{|l|}{ Usa chupeta } \\
\hline Sim & 3,42 & 4,20 & 6,17 & 4,63 \\
\hline Não & 18,61 & 12,90 & 14,39 & 14,90 \\
\hline$p$ & $<0,002$ & $<0,001$ & $<0,001$ & $<0,001$ \\
\hline \multicolumn{5}{|l|}{ Usa mamadeira } \\
\hline Sim & 6,25 & 6,32 & 6,56 & 6,59 \\
\hline Não & 23,00 & 24,00 & 24,00 & 24,00 \\
\hline$p$ & 0,041 & 0,002 & $<0,001$ & $<0,001$ \\
\hline \multicolumn{5}{|c|}{ Características pré-natais } \\
\hline \multicolumn{5}{|c|}{ Número de filhos } \\
\hline Um & 6,51 & 5,51 & 4,87 & 6,11 \\
\hline Dois ou mais & 6,82 & 7,06 & 12,04 & 8,40 \\
\hline$p$ & 0,946 & 0,229 & 0,021 & 0,010 \\
\hline \multicolumn{5}{|c|}{ Local do pré-natal } \\
\hline Público & 5,85 & 6,28 & 12,15 & 7,46 \\
\hline Privado & 6,80 & 7,54 & 6,91 & 7,64 \\
\hline$p$ & 0,375 & 0,242 & 0,787 & 0,531 \\
\hline \multicolumn{5}{|c|}{ Orientação sobre aleitamento materno } \\
\hline $\operatorname{sim}$ & 6,77 & 6,23 & 11,89 & 7,31 \\
\hline Não & 6,21 & 8,52 & 9,43 & 7,85 \\
\hline$p$ & 0,418 & 0,110 & 0,901 & 0,617 \\
\hline \multicolumn{5}{|c|}{ Características do parto } \\
\hline \multicolumn{5}{|l|}{ Tipo } \\
\hline Vaginal & 6,94 & 6,97 & 9,11 & 7,48 \\
\hline Cesárea & 6,11 & 5,70 & 14,42 & 7,87 \\
\hline$p$ & 0,167 & 0,459 & 0,249 & 0,526 \\
\hline \multicolumn{5}{|l|}{ Local do parto } \\
\hline Público & 5,34 & 6,18 & 12,15 & 7,20 \\
\hline Privado & 8,01 & 9,74 & 6,99 & 8,11 \\
\hline$p$ & 0,114 & 0,122 & 0,884 & 0,162 \\
\hline \multicolumn{5}{|c|}{ Orientação sobre aleitamento materno } \\
\hline Sim & 6,46 & 6,86 & 11,96 & 7,63 \\
\hline Não & 8,25 & 6,00 & 6,55 & 7,02 \\
\hline$p$ & 0,985 & 0,145 & 0,313 & 0,102 \\
\hline \multicolumn{5}{|c|}{ Características sociodemográficas } \\
\hline \multicolumn{5}{|c|}{ Renda familiar per capita (salário mínimo) } \\
\hline$<1,8$ & 6,74 & 4,45 & 6,67 & 6,84 \\
\hline$\geq 1,8$ & 5,93 & 7,15 & 11,79 & 7,84 \\
\hline$p$ & 0,539 & 0,038 & 0,638 & 0,191 \\
\hline \multicolumn{5}{|l|}{ Idade materna } \\
\hline$<30$ anos & 6,61 & 6,19 & 9,30 & 7,01 \\
\hline$\geq 30$ anos & 6,52 & 9,39 & 12,41 & 9,74 \\
\hline$p$ & 0,882 & 0,405 & 0,110 & 0,081 \\
\hline
\end{tabular}

continua 
conclusão

Tabela 2

Mediana de tempo de aleitamento materno (meses) nos diferentes Grupos Sociais Homogêneos, segundo características infantis, maternas (pré-natal e parto) e sócio-demográficas familiares. Itupeva, SP, 2001.

\begin{tabular}{|c|c|c|c|c|}
\hline \multirow[t]{2}{*}{ Variáveis } & \multicolumn{3}{|c|}{ Grupos Sociais Homogêneos } & \multirow[t]{2}{*}{ Total } \\
\hline & GSH1 & GSH2 & GSH3 & \\
\hline \multicolumn{5}{|c|}{ Escolaridade mãe (anos) } \\
\hline Até 3 & 3,00 & 6,49 & 15,11 & 11,72 \\
\hline $4-11$ & 6,67 & 6,79 & 9,38 & 7,38 \\
\hline 12 e mais & 2,75 & 9,50 & 8,50 & 8,60 \\
\hline$p$ & 0,968 & 0,772 & 0,651 & 0,833 \\
\hline \multicolumn{5}{|l|}{ Situação conjugal } \\
\hline Com companheiro & 7,20 & 6,76 & 12,06 & 8,00 \\
\hline Sem companheiro & 3,30 & 7,02 & 4,00 & 4,48 \\
\hline$p$ & 0,059 & 0,772 & 0,382 & 0,033 \\
\hline \multicolumn{5}{|l|}{ Trabalho materno } \\
\hline Sim & 7,23 & 4,38 & 6,77 & 6,11 \\
\hline Não & 6,54 & 7,25 & 11,47 & 7,93 \\
\hline$p$ & 0,516 & 0,066 & 0,853 & 0,065 \\
\hline \multicolumn{5}{|l|}{ Escolaridade pai (anos) } \\
\hline Até 3 & - & 16,00 & 24,00 & 24,00 \\
\hline $4-11$ & 6,84 & 6,99 & 7,61 & 7,44 \\
\hline 12 e mais & 23,00 & 6,00 & 19,00 & 8,43 \\
\hline$p$ & 0,420 & 0,196 & 0,009 & 0,006 \\
\hline
\end{tabular}

*Teste de Wilcoxon; GHS=Grupos Sociais Homogêneos; AM=aleitamento materno.

Tabela 3

Valores do Hazard Ratio e intervalo de confiança obtidos no modelo de Regressão de Cox para as variáveis finais selecionadas. Itupeva, São Paulo, 2001.

\begin{tabular}{lccc}
\hline Variáveis & Hazard Ratio & (IC95\%) & $p$ \\
\hline $\begin{array}{l}\text { Ordem de nascimento } \\
1^{\circ}\end{array}$ & 1,0 & - & \\
$2^{\circ}$ em diante & 0,65 & $(0,46-0,93)$ & 0,018 \\
Uso de chupeta & 3,05 & $(2,13-4,37)$ & $<0,001$ \\
$\quad$ Sim & 1,0 & - & \\
Não & & & \\
Uso de mamadeira & 17,13 & $(4,20-69,86)$ & $<0,001$ \\
$\quad$ Sim & 1,0 & - & \\
Não & & & \\
\hline
\end{tabular}




\section{Discussão}

A duração mediana do AM observada em Itupeva foi de 7,2 meses, valor discretamente menor do que os 7,6 meses encontrados para o Brasil em 2006.18 Investigações realizadas em municípios e/ou estados brasileiros mostram medianas de AM bastante diversas, porém com tendência de aumento, sobretudo nas últimas duas décadas. Na Grande São Paulo, em 1987, a mediana da duração do AM era de apenas 4,3 meses, ainda assim, maior que em 1981 (3,0 meses). ${ }^{19}$ No município de Pelotas, Estado do Rio Grande do Sul, a mediana em 1993 era de 4,0 meses. ${ }^{20} \mathrm{Na}$ década seguinte, constatou-se 6,7 meses na Região Nordeste do Brasil. ${ }^{21}$

Os resultados do presente estudo, avaliando a prática do aleitamento materno segundo perfis de reprodução social (GSH), indicam que as crianças do GSH3 apresentaram a maior mediana de duração do $\mathrm{AM}$, da mesma forma que, no momento da entrevista, se observou maior proporção de amamentação entre as crianças desse grupo. 11

Apesar da tendência de maior duração do aleitamento materno no grupo que integrava as crianças socialmente mais excluídas, a diferença não foi estatisticamente significante, indicando que essa prática não se associava com as condições sociais e econômicas das famílias estudadas.

Para melhor entender essa tendência, foram analisados o comportamento das variáveis maternas e infantis nos diferentes GSH e a influência de tais características na duração do aleitamento materno, considerando a especificidade de cada GSH. A reprodução social, explicitada pelos GSH, não foi indicada como variável associada à duração do AM na análise bivariada. Apesar disso, foi inserida no modelo de análise múltipla como forma de melhor ajuste, porém também não se mostrou significante nessa etapa. Assim, os resultados evidenciaram que a prática do AM em Itupeva não era diferente entre os GSH, da forma como esses foram construídos. Há, pois, que se considerar a possibilidade de que a metodologia utilizada para a construção dos GSH não tenha selecionado indicadores e pontos de corte adequados para a construção dos grupos sociais.

Em Salvador, Estado da Bahia, a duração mediana do aleitamento materno total também não foi diferente em crianças menores de 24 meses de famílias com condições de vida elevada e muito baixa, porém as crianças de famílias com piores condições de vida apresentavam 2,5 vezes mais chances de serem desmamadas precocemente em relação àquelas que tinham melhores condições de sobrevivência. 6
Mesmo não se constatando diferença significante nas curvas de sobrevida entre os três GSH, não se pode desconsiderar ou subestimar o papel da determinação social nos perfis de AM, visto que as variáveis que interferiram nas curvas de cada GSH refletem a reprodução social desses grupos.

Em Itupeva, a cobertura de atendimento prénatal $(99,6 \%)$ não foi diferente entre os GSH, e representa o percentual encontrado na região Sudeste, onde apenas $0,2 \%$ das mulheres não haviam realizado nenhuma consulta de pré-natal em 2006.18 Embora a maioria das mães tenha sido assistida pelo serviço público - tanto no pré-natal como no parto sua proporção no GSH3 foi significantemente maior em relação aos demais grupos. Essa diferença reflete a precariedade da inserção social das mães desse grupo, confirmada pela maior proporção de famílias com menor RFPC, menor escolaridade materna e paterna, menor inserção no mercado de trabalho e maior número de filhos. Destaca-se, entretanto, que somente metade das mulheres entrevistadas referiu ter recebido informações ou incentivo para o AM durante as consultas de pré-natal, proporção menor que a encontrada em outros inquéritos sobre o AM.22,23 No atendimento ao parto, orientações sobre o AM atingiu quase $90 \%$ das mulheres, não havendo diferença significante entre os grupos. Essa proporção foi mais elevada do que a observada em Belo Horizonte, Estado de Minas Gerais (58,6\%). 23 Em Salvador, constatou-se que a orientação sobre AM na maternidade variava significantemente com as condições sociais e econômicas da família, porém mesmo entre as mães de muito baixo nível socioeconômico que apresentaram os menores percentuais, a taxa foi de $65,4 \%{ }^{6}$

A resultante desinformação das mães poderia justificar em parte a elevada frequência do uso da chupeta $(53,3 \%)$ e da mamadeira $(78,9 \%)$, percentuais que se mostraram, respectivamente, similar $(55,4 \%)$ e maior $(48,8 \%)$ ao encontrado em Feira da Santana, Estado Bahia. 24 É importante salientar que a utilização da chupeta e da mamadeira foi comum aos três GSH e essas variáveis foram as únicas que mostraram associação com a duração do AM nos três grupos sociais. Esse resultado expressa a necessidade contínua de se enfatizar para as mães e profissionais de saúde os efeitos prejudiciais desses hábitos sobre a amamentação. ${ }^{25} \mathrm{~A}$ chupeta tem forte associação com desmame precoce, de forma que crianças que não a usam apresentam maior prevalência e duração do aleitamento materno, quando comparadas às que a utilizam.26,27 Entretanto, seu uso ainda se constitui em um hábito cultural importante em nosso meio, com frequência 
de $53,6 \%$ entre crianças desmamadas e $27,6 \%$ entre as que ainda mamam. ${ }^{17}$

Renda familiar e escolaridade materna, que classicamente têm sido associadas às taxas de AM, não interferiram na prática da amamentação em Itupeva, a não ser no GSH2, o mais heterogêneo dos grupos sociais, por congregar famílias com FT ou FV inadequadas. O GSH3, o mais precariamente inserido socialmente, foi também o mais exposto à interferência de variáveis sócio-demográficas (ordem de nascimento, número de filhos e escolaridade paterna), variáveis que se mostraram diferenças significantes nesse grupo quando comparado aos demais. Entretanto, tais associações desapareceram na análise ajustada, exceto ordem de nascimento.

Constatou-se menor duração mediana da amamentação entre as mães de $1^{\circ}$ filho e de apenas um filho, quando comparadas àquelas com dois ou mais filhos. Considerando que a amamentação não é apenas um ato natural e fisiológico, mas social e historicamente determinado, ${ }^{9}$ a experiência anterior e o aprendizado figuram como determinantes importantes das taxas de AM.

De uma forma geral, os dados encontrados indicaram que a duração do AM total é expressivamente menor do que aquela recomendada pela OMS, que indica sua manutenção até pelo menos os dois anos de idade. $28 \mathrm{O}$ conhecimento sobre as tendências locais do padrão de amamentação é fundamental, pois permite avaliar os serviços de saúde e embasar mudanças e ajustes necessários às práticas de promoção e incentivo ao aleitamento materno. 5

Mesmo reconhecendo as possíveis limitações técnicas dos indicadores compostos usados na construção dos grupamentos sociais, o embasamento teórico que a fundamentou tem permitido avançar na explicação de como cada formação social cria um padrão de fortalecimento e desgaste que determina seu processo de saúde-doença. Assim, embora a construção dos perfis sociais se constitua em um artefato técnico que auxilia no entendimento da realidade, por sua própria natureza, há limites na correspondência entre perfis de reprodução social e padrão de aleitamento materno, aqui considerado inserido no perfil de saúde-doença. Isto porque os determinantes do processo saúde-doença são complexos e interligados, sendo a concepção desse processo e das formas de cuidado nele envolvidas - entre elas o aleitamento materno - embebidos à cultura e aos próprios valores e anseios individuais, fato já seguidamente reiterado por estudos sobre as causas do desmame precoce.

Dessa forma, mesmo não se constatando associação entre duração do aleitamento materno e os grupos sociais estudados, não há como se desconsiderar a relevância dos determinantes sociais nas ações de promoção do AM, que necessariamente devem incluir esclarecimentos sobre os efeitos prejudiciais do hábito de oferecer chupetas e mamadeiras. Enquanto projeto de intervenção imediata, os resultados deste estudo têm servido para redirecionar as ações desenvolvidas no município, com vistas a aumentar a duração do AM. Nesse sentido, tem se buscado ampliar as oportunidades de orientação, ora presentes não apenas no pré-natal e parto, mas também no puerpério e no atendimento à criança, incluindo maior atenção às primíparas, além da abordagem da influência negativa do uso de mamadeira e chupeta no estabelecimento efetivo do aleitamento materno.

\section{Referências}

1. American Academy of Pediatrics. Work Group on Breastfeeding. Breastfeeding and the use of human milk. Pediatrics. 2005; 115: 496-506.

2. Perez-Escamilla R. Patrones de la lactancia natural en América Latina y el Caribe. Bol Oficina Sanit Panam. 1993; 115: 185-93.

3. Coulibaly R, Séguin L, Zunzunegui MV, Gauvin L. Links between maternal breast-feeding duration and Québec infants' health: a population-based study. Are the effects different for poor children? Matern Child Health J. 2006; 10: $537-43$.

4. Giugliani ERJ, Issler RMS, Kreutz G, Meneses C, Justo EB, Kreutz V, et al. Breastfeeding pattern in a population with different levels of poverty in Southern Brazil. Acta Paediatr. 1996; 85: 1499-500.
5. Pedroso GC, Puccini RF, Silva EMK, Silva NN, Alves MCGP. Prevalência de aleitamento materno e introdução precoce de suplementos alimentares em área urbana do Sudeste do Brasil, Embu, SP. Rev Bras Saude Matern Infant. 2004; 4: 45-58.

6. Oliveira LPM, Assis AMO, Gomes GSS, Prado MS, Barreto ML. Duração do aleitamento materno, regime alimentar e fatores associados segundo condições de vida em Salvador, Bahia, Brasil. Cad Saúde Pública. 2005; 21 : 1519-30.

7. Buralli KO. Aleitamento materno: o social desfigurado [tese]. São Paulo: Faculdade de Saúde Pública da Universidade de São Paulo; 1986.

8. Faleiros FTV, Trezza EMC, Carandina L. Aleitamento materno: fatores de influência na sua decisão e duração. Rev Nutr. 2006; 19: 623-30. 
9. Almeida JAG, Novak FR. Amamentação: um híbrido natureza-cultura. J Pediatr (Rio J). 2004; 80 (5 suppl): S119-25

10. Laurell AC. A saúde-doença como processo social. In: Nunes EP. (org). Medicina social: aspectos históricos e teóricos. São Paulo: Global; 1983. p.134-58.

11. Minagawa AT, Oliveira IMV, Fujimori E, Laurenti E, Montero RMJM. Perfil do aleitamento materno em menores de 2 anos na cidade de Itupeva, SP, Brasil. Arch Latinoam Nutr. 2005; 55: 132-9.

12. Fundação Sistema Estadual de Análise de Dados. Informações dos Municípios Paulistas. 2001. [2001 Maio 15] Disponível em: http://www.seade.gov.br

13. Fundação Instituto Brasileiro de Geografia e Estatística. Cadastro de cidades e vilas do Brasil e malha municipal digital. 1997. [2001 Maio 15] Disponível em: http://www.ibge.gov.br/cidades

14. Monteiro CA, Szarfarc SC, Mondini L. Tendência secular da anemia na infância na cidade de São Paulo (1984-1996). Rev Saúde Pública. 2000; 34 (6 supl): 62-72.

15. Queiroz VM, Salum MJL. Operacionalizando a heterogeneidade do coletivo na releitura da categoria de reprodução social. São Paulo: EEUSP; 1997.

16. Ortega LDS, Oliveira IMV, Fujimori E, Biagolini REM, Minagawa AT. Reproducción social de las familias de niños menores de 7 años. Rev Escola Enf USP. 2005; 39: 333-42.

17. Organización Panamericana de la Salud. Organización Mundial de la Salud. Indicadores para evaluar las prácticas de lactancia materna. Genebra: OMS/CED/SER/91.14; 1991.

18. Brasil. Ministério da Saúde. PNDS 2006. Pesquisa Nacional de Demografia e Saúde da Criança e da Mulher. Brasília, DF; 2008. [acesso em 16 fev 2009] Disponível em: http://bvsms.saude.gov.br/bvs/pnds/img/relatorio_final_pn ds2006.pdf

19. Rea MF, Berquó ES. Impact of the Brazilian national breast-feeding programme on mothers in Greater São Paulo. Bull World Health Organ. 1990; 68: 365-71.

Recebido em 6 de agosto de 2008

Versão final apresentada em 22 de setembro de 2009

Aprovado em 23 de outubro de 2009
20. Horta BL, Olinto MTA, Victora CG, Barros FC, Guimarães PRV. Amamentação e padrões alimentares em crianças em duas coortes de base populacional no sul do Brasil: tendências e diferenciais. Cad Saúde Pública. 1996; 12 (Supl 1): 43-8.

21. Lima TM, Osório MM. Perfil e fatores associados ao aleitamento materno em crianças menores de 25 meses da Região Nordeste do Brasil. Rev Bras Saúde Matern Infant. 2003; 3 : 305-14.

22. Vasconcelos MGL, Lira PIC, Lima MC. Duração e fatores associados ao aleitamento materno em crianças menores de 24 meses de idade no estado de Pernambuco. Rev Bras Saúde Matern Infant. 2006; 6: 99-105.

23. Figueiredo LMH, Goulart EMA. Análise da eficácia do Programa de Incentivo ao Aleitamento Materno em um bairro periférico de Belo Horizonte (Brasil): 1980/1986/1992. J Pediatr (Rio J). 1995; 71: 203-8.

24. Vieira GO, Glisser M, Araújo SPT, Sales AN. Indicadores do aleitamento materno na cidade de Feira de Santana, Bahia. J Pediatr (Rio J). 1998; 74: 11-6.

25. Lamounier JA. O efeito de bicos e chupetas no aleitamento materno. J Pediatr (Rio J). 2003; 79: 284-6.

26. Vieira GO, Almeida JAG, Silva LR, Cabral VA, Santana Netto PV. Fatores associados ao aleitamento materno e desmame em Feira de Santana, Bahia. Rev Bras Saúde Matern Infant. 2004; 4: 143-50.

27. Soares MEM, Giugliani ERJ, Braun ML, Salgado ACN, Oliveira AP, Aguiar PR. Uso de chupeta e sua relação com o desmame precoce em população de crianças nascidas em Hospital Amigo da Criança. J Pediatr (Rio J). 2003; 79: 309-16.

28. World Health Organization. The optimal duration of exclusive breastfeeding. Note for press $\mathrm{n}^{\circ} 7,2001$. [2001 Maio 15] Disponível em: http://www.who.int/inf-pr2001/en/note2001-07.html 